\title{
Concepciones de investigación y realización académica en las ciencias sociales colombianas: los investigadores de la Universidad de Antioquia y la Universidad de los Andes*
}

DOI: https://doi.org/10.18046/recs.i31.3484

\author{
Concepts of Research and Academic Achievement in \\ the Colombian Social Sciences: Researchers from the University \\ of Antioquia and the University of the Andes
}

\author{
Rodolfo Masías ${ }^{*}$ \\ Universidad de los Andes (Bogotá, Colombia)
}

\section{Carlos Andrés Aristizábal-Botero***}

Universidad de Antioquia (Medellín, Colombia)

\footnotetext{
* Este artículo es resultado del proyecto Productos de investigación en ciencias sociales, artes y humanidades: concepciones de valoración y mecanismos de validación, evaluación e impacto, financiado en convocatoria conjunta por la Universidad de los Andes y la Universidad de Antioquia. Artículo de investigación recibido el 21.03.2019 y aceptado el 21.02.2020.

** Doctor en Ciencias Sociales con especialidad en Sociología por El Colegio de México (México). Profesor asociado III de la Facultad de Ciencias Sociales de la Universidad de los Andes (Colombia).Correo electrónico: rmasias@uniandes. edu.co ORCID: https://orcid.org/oooo-ooo3-2512-6358

*** Candidato a doctor en Ciencias Sociales y Humanas de la Universidad Nacional de Colombia, sede Bogotá (Colombia). Profesor del programa de Sociología de la Universidad de Antioquia (Colombia).Correo electrónico: carlos.aristizabal@ udea.edu.co ORCID: https://orcid.org/oooo-ooo1-5821-4878
} 


\section{Cómo citar/How to cite}

Masías, Rodolfo; Aristizábal-Botero, Carlos Andrés (2020). Concepciones de investigación y realización académica en las ciencias sociales colombianas: los investigadores de la Universidad de Antioquia y la Universidad de los Andes. Revista CS, 31, 385-412.

https://doi.org/10.18046/recs.i31.3484 


\section{Resumen}

En esta investigación se dilucidan, como tipos ideales, concepciones sobre la vida y la situación social de investigador. Más importante, concepciones sobre la valoración de lo que se denomina realización académica. Esta es una categoría relacionada con la culminación (y comunicación) de procesos de investigación, lo que se ha generalizado como producción de productos. Las concepciones tipificadas versan sobre lo que es y debe ser un investigador, la investigación en ciencias sociales y la idea misma de realización. Además, se refieren a cómo son y deben ser valorados y evaluados los resultados de la actividad académica. Se sugiere, como hallazgo, la coexistencia de diversas concepciones sobre lo que es y se hace en las ciencias sociales. La investigación estribó en entrevistas a profundidad a profesores de programas de ciencias sociales pertenecientes a la Universidad de Antioquia y a la Universidad de los Andes, en Colombia.

\section{PALABRAS CLAVE:}

concepciones del investigador, producción científica, valoración de productos de investigación, sociología del conocimiento

This research elucidates, as ideal types, conceptions about life and the social situation of the researcher. More importantly, conceptions about the value of what is called academic achievement. This is a category related to the culmination (and communication) of research processes, which has become generalized as product production. Typified conceptions deal with what a researcher is and should be, social science research and the very idea of achievement. Furthermore, they refer to how the results of academic activity are and should be valued and evaluated. It is suggested, as a finding, the coexistence of diverse conceptions about what is and what is done in the social sciences. The research was based on in-depth interviews with professors from social science programs at the University of Antioquia and the University of the Andes in Colombia.

\section{KEYWORDS:}

Researcher's Conceptions, Scientific Production, Evaluation of Research Products, Sociology of Knowledge 



\section{Objeto de estudio y objetivo de la investigación}

Planteado a un nivel teórico, el objeto de estudio de esta investigación involucra tres conceptos: el de régimen de la ciencia, el de concepción o concepciones del investigador social y el de realización académica o científica, que implica su valoración. El objetivo es constatar la existencia de estas concepciones, su diversidad, y a la vez comenzar a delinear sus rasgos, contenidos típicos y la concordancia o no con las características actuales de lo que denominamos régimen de la ciencia.

El régimen del que tratamos es, ciertamente, un régimen histórico: es el conjunto de reglas y valores formalizados, regulados y transmitidos por las instituciones que conforman, para nuestro campo de observación empírica, el sistema nacional de ciencia y tecnología colombiano, donde Colciencias es la principal institución reguladora ${ }^{1}$. Esta, por medio de políticas científicas, vincula al sistema nacional con el régimen global de conocimiento. La tendencia de las políticas de Colciencias ha sido la de reproducir lo que se ha establecido como regular en el régimen global'. Por su parte, concepciones es un compuesto conceptual que conjuga ideas, creencias, opiniones y conocimientos. Así, la reconstrucción de concepciones que aquí se presenta da cuenta de las elaboraciones subjetivas sobre lo que son y hacen unos investigadores sociales que desarrollan su actividad académica e investigativa como docentes vinculados a instituciones universitarias. Son unas instituciones que participan del régimen actual, por lo cual expresan, en sus reglamentaciones internas, como normas a cumplir, unos específicos comportamientos científicos y académicos.

Definiendo la concepción del propio régimen respecto de lo que es investigar, lo que es un investigador y la realización académica o científica, esta concepción se manifiesta con unas características formas de percibir la ciencia y su papel, así como la naturaleza deseable y la razón de ser de la práctica de la investigación y de la identidad de investigador (en esta concepción, las ciencias sociales son subsumidas a un tal conjunto unitario de la ciencia, pues no se las considera distintas de las ciencias naturales) (Consejo Nacional de Política Económica y Social, 2015).

1. "Colciencias es el Departamento Administrativo de Ciencia, Tecnología e Innovación. Promueve las políticas públicas para fomentar la CTI en Colombia. Las actividades alrededor del cumplimiento de su misión implican concertar políticas de fomento a la producción de conocimientos, construir capacidades para CTI, y propiciar la circulación y usos de los mismos para el desarrollo integral del país y el bienestar de los colombianos" (Colciencias, 2019). Sobre el papel de Colciencias, ver Jiménez (2015).

2. Esto no significa que otros agentes no estipulen el régimen actual. En la expansión e instauración de este régimen participan universidades, centros de investigación, empresas privadas y, por supuesto, otros actores estatales como el Ministerio de Educación y Cultura. Como sabemos, tampoco se trata de una situación original o particular de Colombia. La implantación del régimen actual tiene los visos de una experiencia histórica global. 
Particularmente, también esta concepción manifiesta una elaboración muy concreta sobre la forma como debe culminar un proceso de investigación, y cómo y por quién deben ser valorados y validados los resultados que consuman estas experiencias de conocimiento (Lux; Pérez, 2017). La ciencia ahí es más una actividad de tipo productivo, es vista como una fuerza productiva, en términos marxistas (Marcuse, 1993), por lo que es ideal la ciencia que accede al mundo de la tecnología y estrecha vínculos con el mundo empresarial (Etzkowitz; Leydesdorff, 1997), mientras se insta a que el investigador, por su parte, se comprenda a sí mismo como un productor de productos. No es, o no parece ser en esta concepción, un sujeto cognitivo cuyo fin vital es el conocimiento (Masías, 2014). Esta concepción, al ver al investigador como un sujeto productivo, eleva cómo deber ser la maximización de su actividad o su intensidad de trabajo, de modo que no sea solo un sujeto que produce, sino altamente productivo.

Una consecuencia de la concepción que analizamos es que se crea que los llamados productos del investigador deban encontrar su valor más en la constitución interna del producto en conexión con el mundo social y político. La concepción fomenta, así, la idea de que se aporta produciendo en cantidad, no importando si esa producción resulte útil o conlleve unas consecuencias socialmente significativas. Pareciera que el valor de una realización científica o investigativa importara solo, o más, por su validez que por su valor; entendiendo validez como el cumplimiento, en el proceso de producción del producto, de los protocolos que lo hacen reconocible como un resultado académico; y su valor, como su aporte, significado, trascendencia o su incidencia en la historia y la sociedad.

Sobre el tercer concepto que contiene nuestro objeto de estudio, conviene ser más exhaustivos: realización académica o científica. Este concepto lo acuñamos como una alternativa al significado que subyace al uso de producción y producto, como denominaciones para definir los resultados de una investigación y su comunicación. Realización académica captura la diversidad de formas materiales en las cuales se expresan los resultados de la investigación social. Es una alternativa para enfrentar la reducción de la actividad de investigación a un proceso meramente productivo de consumación de una sola finalidad, concretada solo en determinados objetos como los artículos, considerados el producto final de toda actividad comunicativa de carácter científico, como lo han expresado, entre otros, Callon, Courtial y Penan (1995). Realización es una noción, además, que ensancha el campo de la observación y de la interpretación, tanto de lo que hace el investigador que está inmerso en un proceso de conocimiento como de lo que busca conseguir al final de este. Sin embargo, la idea misma de realización representa bien la situación respecto a los procesos de investigación que, de alguna manera, no terminan, sino que son experiencias 
continuadas de elaboración intelectual que, si bien tienen altos de concreción, no necesariamente estos constituyen la coronación absoluta de tales procesos. Un componente importante que quiere recuperar esta conceptualización es la satisfacción del investigador con los resultados de sus trabajos y con el ideal de ver sus frutos con utilidad y repercusión en las esferas de la sociedad.

La concepción en cuestión, que hemos denominado del régimen, se ha hecho más patente y hegemónica durante los últimos diez años, aproximadamente (Villaveces; Forero-Pineda, 2007). Se ha convertido, en verdad, en el punto de referencia, no solo axiológico, sino normativo e institucional, para todo aquel que sea o que quiera pertenecer al mundo de la investigación y de la academia de las ciencias sociales. Su cada vez mayor presencia, su naturalización y normalización dejan la impresión no solo de imperar, sino de conformar, ya, una especie de consenso de concepción entre los académicos. Es decir, unas ciencias sociales todas que reconocerían y legitimarían esta visión. Es una duda sobre esta realidad la que motiva la investigación que aquí presentamos. Porque, ciertamente, cabe preguntarse: ¿existe tal consenso de concepción? La concepción referida, ¿es la única concepción existente en la academia de las ciencias sociales en Colombia y, específicamente, en la Universidad de Antioquia y la Universidad de los Andes? Y si hay más concepciones, ¿qué ven?, ¿qué afirman?, ¿qué percepciones han desarrollado sobre las cualidades de la concepción del régimen?, ¿qué sostienen sobre lo que es investigar, un investigador y su realización académica?

La problemática que da origen a esta investigación está ligada con el fenómeno que Gómez-Morales (2018) ha denominado como cultura de la auditoría y que se ha convertido en la manera de promover una determinada forma de entender la actividad investigativa, caracterizada por el eficientismo y la productividad. La lógica regulativa de esta cultura se ha profundizado desde principios de la segunda década del siglo XXI, cuando en Colombia se estructuraron modelos evaluativos vinculados con modelos de incentivos (Gómez-Morales, 2017). Esta cultura ha incorporado patrones hipernormativos de la agenda científica internacional en el trabajo académico local, como lo presenta Gómez-Morales (2015).

Autoras como Gill (2014) consideran que la institucionalización de estos modelos de vigilancia académica, al intensificar la demanda de eficiencia y productividad, generan como efecto la precarización de las condiciones de trabajo de los investigadores, así como transformaciones subjetivas que afectan otras relaciones como las sociales y familiares. Guzmán Tovar (2019) nos presenta una situación similar en el caso de investigadores sociales latinoamericanos: la aceleración de la actividad investigativa, confundida con eficiencia y productividad, ha estructurado una matriz de acción que se replica en la organización de las diferentes esferas de su 
vida. La subjetividad del investigador se ha visto afectada por una trasformación normativa que no necesariamente se corresponde con las concepciones sobre la naturaleza de su labor.

Por su parte, trabajos como los de Santos $(2012 ; 2015)$ precisan las consecuencias y malestar que produce que la lógica productivista de la actividad científica haya establecido que el tipo por excelencia de realización sea el paper. Es toda una concepción de la realización académica cuya puesta en práctica, como lo muestra Masías (2014), ha ocasionado unos nuevos fenómenos de diferenciación y desigualdad social en las comunidades académicas. Los investigadores poco productivos terminan por no reunir las condiciones que les permitan participar de un sistema científico que margina.

Consideramos que la actividad científica no se restringe a una productividad de artículos, como tampoco podemos basar el conocimiento de las dinámicas sociales y políticas de la academia y la ciencia exclusivamente en este fenómeno (Vasen; Lujano-Vilchis, 2017). Hay otras formas de realización académica que permiten observar no solo esas expresiones que dan cuenta de la producción del investigador, sino que también dan cuenta de las subjetividades que los orientan en aquello que entienden como trabajo académico. Vessuri (2013), al preguntarse sobre quién es el científico social en el siglo XXI, no encuentra una respuesta que capte las posibles identidades, pero sí se pregunta si es el Estado el único lugar desde donde se realizan las ciencias sociales. Afirma que para que no fuera así, sería necesario "reconocer tradiciones particulares, así como sus propias obras culturales, epistemologías y marcos teóricos, culturas de la ciencia y lenguajes de pensamiento, al igual que sitios de producción y transmisión del conocimiento" (Vessuri, 2013: 225).

En consecuencia, este trabajo aporta a la reflexión sobre la subjetividad o, si se quiere, las subjetividades, de algunos investigadores de las ciencias sociales colombianas. Sabemos poco, o intuitivamente, si hemos experimentado un cambio generalizado de concepción; como tampoco si asistimos, más bien, a una diversificación de concepciones que coexisten conflictiva o armónicamente. Por sobre esta aportación, la investigación se justifica porque, en la medida en que efectivamente asistimos a unas grandes transformaciones en el mundo de la ciencia, como lo sostienen, de acuerdo con sus respectivas filiaciones teóricas, De Sousa-Santos (2010; 2011), Brown (2009), Wallerstein (1999; 2005), Cathalifaud (2012) y Kreimer (2006; 2011), hace falta tener unas versiones cada vez más sistemáticas de los cambios en el plano de eso que hemos llamado concepciones. Es una situación en que hay avidez por saber quiénes somos y cómo formamos identidades en este micromundo del conocimiento. 


\section{Diseño metodológico}

Este trabajo es una de las realizaciones de un proyecto de investigación ${ }^{3}$ conjunto entre la Universidad de Antioquia y la Universidad de los Andes. En su aspecto investigativo, y respecto del objeto y objetivo ya mencionados, era interés del proyecto abordar diferentes realidades universitarias. Valga esta explicación para aclarar un poco el contexto sobre el que se tomaron las principales decisiones metodológicas.

Dada la naturaleza del elemento principal del objeto de estudio, como son las concepciones de los sujetos, se convino diseñar una metodología de investigación cualitativa. En efecto, por suponer que la forma más eficaz de acceder a las concepciones es mediante el testimonio de los mismos involucrados, se prefirió como unidad de análisis exclusiva al investigador individual, de quien se estudian sus formas de pensar, a partir de su propio discurso. En tal sentido, la unidad de análisis es también unidad de información o fuente.

Se optó por una estrategia de muestreo teórico. Por ser un estudio sobre dos universidades, la concepción del muestreo partió del principio de representación equivalente y equitativa por cada universidad. Lo que siguió fue precisar aquellas variables que permitieran una selección representativa, como la disciplina, el sexo, la edad y la trayectoria de los investigadores, que funcionaron fundamentalmente como criterios de elección, mas no como variables independientes en el análisis posterior. Así, los casos en que estriba esta investigación representan a las dos universidades, a sus distintos programas de ciencias sociales, a su distribución por género, por edad y por experiencia académica. Estas características están comprendidas en la muestra final compuesta por 41 investigadores en total. Los casos concretos fueron elegidos al azar, entre las listas de la planta de las dos universidades. Las entrevistas se desarrollaron entre marzo y septiembre de 2015, y su análisis, entre 2016 y 2017. Tuvieron una duración promedio de una hora y treinta minutos. La mayor parte de ellas se efectuó en la oficina de los profesores. El Cuadro 1 permite una idea del contenido de la guía de entrevista utilizada, pero también, dar paso a la explicación de la estrategia de análisis e inferencia en esta investigación.

El análisis se llevó a cabo con el programa Atlas.Ti. Con las categorías que aparecen en el Cuadro 1, se procedió a categorizar la información procedente de las entrevistas. Este trabajo permitió encontrar los segmentos de discurso más significativos entre cada testimonio. La categorización llevó a la creación de grupos discursivos que facilitaron reconstruir unas concepciones, tal como era el cometido. El análisis que se presenta a continuación muestra los resultados de un ejercicio descriptivo de

3. "Productos de investigación en ciencias sociales, artes y humanidades: concepciones de valoración y mecanismos de validación, evaluación e impacto”. 
CUADRO 1 $\mid$ Estructura de análisis

\section{Opinión general sobre la}

situación de su disciplina

\begin{tabular}{|c|c|}
\hline \multirow{3}{*}{$\begin{array}{l}\text { Concepción del valor de un producto o una } \\
\text { creación }\end{array}$} & Residencia del valor de productos o creaciones \\
\hline & Medidas a tomar \\
\hline & Productos o creaciones valiosas \\
\hline \multirow{2}{*}{$\begin{array}{l}\text { Aspectos o momentos de un proceso de } \\
\text { valoración }\end{array}$} & $\begin{array}{l}\text { Momentos esenciales e insustituibles. Reacción } \\
\text { frente al diagrama del proyecto }\end{array}$ \\
\hline & Cambio en el proceso: experiencia o trayectoria \\
\hline \multirow{3}{*}{$\begin{array}{l}\text { Formas de valoración actual en el campo } \\
\text { específico }\end{array}$} & Tendencias de valoración según producto o creación \\
\hline & Agentes de valoración \\
\hline & Dispositivos de valoración \\
\hline \multirow{3}{*}{$\begin{array}{l}\text { Forma de acreditación de un producto o } \\
\text { una creación }\end{array}$} & Explicación de acreditación: valor vs. acreditación \\
\hline & Agentes de acreditación \\
\hline & Dispositivos de acreditación \\
\hline
\end{tabular}

Reconstrucción de la adjudicación de valor y acreditación en el proceso largo que va de la concepción a la consumación de un producto o creación

Momentos existentes en el proceso

\begin{tabular}{|c|c|}
\hline \multirow{4}{*}{$\begin{array}{l}\text { Sentido de la comparación de valor y } \\
\text { valoración y acreditación con la producción } \\
\text { ajena }\end{array}$} & Sentido de equidad \\
\hline & Autovaloración de producción \\
\hline & Juicio sobre otras valoraciones \\
\hline & Valoraciones pérfidas \\
\hline \multirow{4}{*}{$\begin{array}{l}\text { Imaginación de la relación entre espacio } \\
\text { geográfico, producción de conocimiento y } \\
\text { acreditación de un producto }\end{array}$} & Lugares nacionales de productos de valor \\
\hline & Lugares nacionales de acreditación \\
\hline & Lugares internacionales de valor \\
\hline & Lugares internacionales de acreditación \\
\hline
\end{tabular}

Fuente: elaboración propia.

reconstrucción global de concepciones, es decir, de la reconstrucción que se funda en la constatación de patrones que se manifiestan con independencia de cualquier característica o variable en particular, sea la universidad de pertenencia, el género, la disciplina o la edad. Como ya lo hicimos saber, nuestra forma de reconstrucción tiene inspiración en la metodología de los tipos ideales. El lector verá un predominio de la generalización, pero podrá atisbar también singularidades. Una reconstrucción, 
en el espíritu de los tipos ideales, no es otra cosa que la creación de conceptos que puedan dar significado al acontecer. A ellos les subyace un interés fenomenológico, pues el cometido se encuentra en la exploración subjetiva de la experiencia vivida, con la cual expresamos nuestra interpretación.

\section{Concepciones sobre la situación actual de la investigación en ciencias sociales}

Hay una concepción coincidente, una percepción entre los investigadores, acerca de la situación actual de las ciencias sociales. Decir concepción coincidente es hablar de visiones, elaboraciones, relatos o versiones sobre el estado de cosas, que tienden a concordar, a ser expresiones muy semejantes o que propenden a complementarse. Nos llamó la atención, a su vez, que no sean coincidencias forzadas, pues aparecen en las entrevistas no solo espontánea, sino inmediatamente en el discurso de estos sujetos, tal como si fueran unos asuntos de los primeros lugares en una escala vital de preocupaciones. Además, cuando estos nos ofrecieron su visión de la situación de las ciencias sociales, parecían estar hablando de lo que viven intensamente, no hablaron como unos expertos que diagnostican, sin pasiones, una realidad. Esto es importante, porque de lo que dan cuenta es de situaciones que les afectan, al parecer profundamente, para bien o para mal; estando de acuerdo o en desacuerdo con lo que pasa.

La actual -no cabe duda- es una situación de alteración, de cambios intensos y profundos en las ciencias sociales, según aflora de las entrevistas. Son modificaciones de tal magnitud y hondura porque los investigadores tienden a concebir el estado del momento como de transformaciones de unas grandes estructuras, que preocupan porque traen consigo, consecuentemente, unos enormes efectos.

La academia no se puede regir por esos sistemas que son absolutamente pensados con una lógica racional capitalista, con una lógica que no tiene mucho que ver con el conocimiento. Es que la academia, hoy por hoy, está situada con unos regímenes que tienen que ver mucho con la eficiencia, con el capital, con ganar excedentes y esa no es la función de la academia. (Entrevistado 16, comunicación personal, 23.06.2015)

La percepción de conjunto observada es a entender lo que viene ocurriendo como parte de procesos macrosociales, de un carácter global, como si se desplegasen en el plano del funcionamiento de un sistema. Hay una concepción estructural y holística en la reconstrucción subjetiva de la realidad social de las ciencias sociales, como tendencia. Más en concreto, los investigadores hablan, como diría Wallerstein 
(1999; 2005), de una gran reorganización de las estructuras del saber, un cambio, en realidad, del orden que rige todas las actuaciones en el mundo de las ciencias sociales. Tendencial o regularmente, se refieren a estas transformaciones como a un severo proceso de institucionalización en procura, supuestamente, de una consolidación de estos saberes y sus instituciones. Si bien estos cambios responden a unas tendencias globales o mundiales, que geopolíticamente se ubican en los grandes centros académicos de poder y legitimación -especialmente Estados Unidos-, en Colombia han tenido un agente generador crucial: Colciencias. En Colciencias, según las entrevistas, se concentra esta visión sobre el origen más inmediato de las alteraciones que se viven. Es una referencia frecuente e ineludible.

Ese medio social que está internacionalmente, que lo determinan las universidades internacionales y el medio social que está acá. Es decir, que las medidas, los parámetros, son producto. No son medidas abstractas, sino que son productos de cierta guía social. (Entrevistado 40, comunicación personal, 04.09.2015)

La internacionalización de la ciencia promovida por Colciencias no solo ha impactado en la producción de artículos científicos, sino que también ha transferido las pautas y procedimientos del ser y hacer ciencia que son promovidas por agentes globales, como las editoriales propietarias de bases de datos cienciométricas. Así, en los testimonios se resaltan rasgos de la situación, que bien pueden ser tomados como efectos, consecuencias o estragos de la ubicua intervención de tales agentes $\mathrm{y}$ dispositivos del régimen. Las intervenciones se han cristalizado, para algunos, en unas interacciones de lucha intensa entre los actores involucrados; a raíz de los cambios, se vive más conflictivamente, en confrontación con los pares, en excesiva competencia. Las modificaciones han reforzado o han creado marginación y procesos de elitización de nueva generación. Como consecuencia, hay quienes dudan de la existencia de consensos en sus ámbitos de trabajo y de la falta de diálogo: no hay discusión, impera el silencio en la academia. "Claramente hay una jerarquía entre el libro en una editorial internacional, el artículo en una revista indexada internacional. Entonces, como que ya todo esto tiene un valor y es como si todos estuviéramos de acuerdo sobre esto" (Entrevistado 19, comunicación personal, 24.07.2015).

La situación se manifiesta, a juicio de los académicos estudiados, como una pérdida de la perspectiva de sus disciplinas, de alteración del sentido que estos conocimientos acostumbraban a tener, un estado de desorientación, pero, asimismo, de extravío de certezas anteriores, que eran no solo acertadas, sino convenientes a juicio de los investigadores. Cuando menos, es una coyuntura que conlleva a la reflexión sobre el ser de las disciplinas. Un ejemplo de ello es lo que se denomina la pérdida de una postura crítica. 
Esta figura de mediador lo que hace es funcionar en términos muy funcionales a lo que se está requiriendo, y deja un poco de lado como la perspectiva crítica. La Antropología, lo creo yo, es que ha perdido totalmente la perspectiva que tenía. (Entrevistado 4, comunicación personal, 15.08.2015)

Si bien con conceptualizaciones y terminología distintas, nos atreveríamos a decir que los investigadores conciben la situación actual como de una administración de conocimiento por parte del Estado y del entorno dominante del saber. Es una ocasión de imposiciones fuertes, muy compulsivas, de reglas o normas que solo se pueden asumir con extrañeza. En sus versiones, existe arbitrariedad y capricho, pues se establecen unas exigencias que trastocan un orden, pero no se corresponden con las posibilidades reales de los miembros de las ciencias sociales. Hay, entre los sujetos de estudio, quienes se atreven a categorizar el estado del momento como de una pérdida de autonomía del campo académico en Colombia. Estarían sobreviniendo unas consecuencias considerables en la relación entre academia y sociedad: se alejarían y se repelerían. Sea como fuere, los testimonios acusan pérdidas, rupturas, invasiones, restricciones en sus ámbitos correspondientes de acción y trabajo.

¿Será que los de las ciencias naturales nos están controlando esto? Porque a veces da esa impresión. Aunque sí es cierto de que es muy complejo tener un solo estándar para todos, pero pareciera que los estándares los hacen pensando desde ciertas disciplinas y no desde otras disciplinas como son las ciencias sociales. (Entrevistado 23, comunicación personal, 10.08.2015)

También es una percepción compartida entre los investigadores que el que podría denominarse nuevo régimen, nuevo orden o sistema de acción académica, insinúa unas características, unas propiedades dominantes que son la expresión de una concepción hegemónica de proyectar las cosas. Es en el terreno de la investigación donde más habría encarnado esta perspectiva sobre el sentido y la finalidad de las ciencias sociales. La concepción/proyecto que revelan las entrevistas y que sería la que se viene imponiendo, es una donde lo que se entiende por investigación es lo más importante para una supuesta cabal identidad académica, tanto que diluye una concepción/proyecto del académico y la academia donde se complementan la investigación, la docencia y la proyección social.

Los testimonios también coinciden en señalar que en la concepción hegemónica la investigación es concebida de un modo muy particular, que es un fin en sí mismo, donde prima una lógica que no tiene que ver necesariamente con el conocimiento. Así, se menciona frecuentemente una perversión o un trastrocamiento de los valores que habían sustentado la práctica de investigar. El principal trastrocamiento 
es el que concentra la realización investigativa en el hecho puro de publicar; acto que, a su vez, remite a unas normas muy precisas y particulares de hacerlo (como publicar en revistas indexadas o tender a publicar más artículos que libros). Se trata de publicar mucho, de ser sumamente productivo. Y se trata de publicar para un mercado que es el que decide los destinos de la investigación en las ciencias sociales. En esta concepción hegemónica que retratan los testimonios, se trata, como una norma, de cumplir sin tapujos con las exigencias de comercialización de los llamados productos de investigación. "El producto académico entra en unas exigencias como mercancía, en un mercado o en un nicho del mercado. Y yo creo que eso es uno de los efectos de las formas actuales, es inescapable" (Entrevistado 10, comunicación personal, 21.05.2015).

Percibimos que esto que está ocurriendo con la investigación crea sentidas preocupaciones, pues estos académicos son capaces de atisbar unas consecuencias inconvenientes. El hecho de que los criterios de mercado se hayan generalizado como ideales, y que se deba responder para investigar y publicar a sistemas de normas cada vez más refinados y técnicos (muchos de ellos del contexto de las ciencias naturales) trae como estragos que estas prácticas se vuelvan tremendamente difíciles sin necesidad, que se restrinja la creatividad y se pierda libertad de pensamiento y acción. Como lo evidencian Nupia (2013) y Gómez-Morales (2015), el sistema de ciencia y tecnología colombiano se encuentra altamente influenciado por modelos extranjeros, así como por una injerencia de la agenda científica internacional, situación que se refleja en los testimonios como una suerte de conciencia esquizofrénica al tener que estar estandarizado internacionalmente y tener, al mismo tiempo, la obligación de crear comunidad académica propia.

Yo creo que hay algo (...) que tiene que ver con el conflicto que le estoy diciendo. (...) Entre estar estandarizado internacionalmente y crear comunidad científica propia. Y es que yo entiendo que la universidad, o cualquier tipo de institución, les dé importancia a los estándares internacionales precisamente porque se trata de las personas que ya evaluaron. Obviamente, la universidad no puede ponerse a leer lo que yo afirmo, sino que tiene que depender mucho de lo que las demás personas dicen. Pero sí es importante que miremos, dado que nosotros entramos en la comunidad científica. Que tengan en cuenta exactamente a los que participan de la comunidad. Cuáles son los criterios que estamos aplicando. (Entrevistado 40, comunicación personal, 04.09.2015) 


\section{Concepciones sobre las formas de valoración actual de la realización académica}

Como era interés del estudio, los investigadores proveyeron sus conceptos acerca de las formas de valoración de lo que en este proyecto llamamos realización académica. Durante las entrevistas, el discurso de los investigadores se explayó simultáneamente entre el diagnóstico, la crítica y el ideal. Al explicar su percepción sobre las maneras predominantes de valorizar, muchos no pudieron evitar confesar sus preferencias más íntimas. Este tema, como el anterior relacionado con la situación actual de las ciencias sociales, moviliza la crítica y los deseos de cambio en el investigador, que habla natural y muy subjetivamente de lo que ocurre en su cotidianidad. Al tiempo, y seguramente por ello, fue posible constatar un discurso muy interior, profundo, resultado, al parecer, de una continua reflexión sobre el tema. Porque, también, por lo visto, para el grueso de sujetos estudiados, la valoración actual de los productos académicos es un problema latente y de atención inmediata.

Pero las formas de valoración de la realización académica no son un asunto que saque a relucir, como tendencia, un claro tipo de concepción consensual o central entre estos investigadores. Podría decirse, por el contrario, que hay unos tipos de concepción, unas formas de ver distintas, que vienen dadas por las preocupaciones que nuclean el discurso sobre el tema. Así, estas concepciones se distinguirían por lo que entienden que serían los principios básicos o rectores que deben sustentar y orientar un proceso de valoración, la adecuación de las formas actuales de valoración a la realidad de las ciencias sociales y la realidad colombiana, al alcance o cobertura de estas formas, y a su finalidad y sentido. Ante este hallazgo, el de unas concepciones divergentes, nos pareció apropiado, analíticamente hablando, tipificar con más precisión cada una de estas. El hallazgo es la existencia de dos clases de concepciones, que contiene una más críticas y radicales y, otra, más relativistas y ponderadoras.

Respecto de los principios básicos o rectores de una valoración, una preocupación que refleja tipos de concepción es la relativa al sujeto de la valoración: ¿es este acto de valorar las realizaciones de los otros, un acto de individuos aislados o de un sujeto colectivo, un actor colectivo? Una de estas concepciones considera que se deben valorar las relaciones académicas (por ejemplo, un libro o un artículo) dentro de un proceso que va más allá de unos pares, es la labor de una entidad que tenga la forma de un comité.

Hay que hacer un trabajo más de comité editorial. Si se quiere dar una identidad a la revista, pues entonces el trabajo va más allá que mandar a dos evaluadores y darle la chance y quizás resaltar algunos artículos que los evaluadores no valoraron tanto. (Entrevistado 19, comunicación personal, 24.07.2015) 
A diferencia de esta concepción, de tipo colectivista social y crítica, la otra que hallamos es la que tiende a justificar y reconocer la inclinación actual de valorar, que deja que el peso de una valoración recaiga en los individuos competentes, denominados pares académicos.

Básicamente, es evaluación por pares. Tú como editor recibes una propuesta, miras que tenga un nivel mínimo adecuado para el perfil de la revista (...), envías el producto a un par de lectores expertos generalmente asociados con el centro, ellos escriben una evaluación, evalúan el argumento, sobre todo. (Entrevistado 11, comunicación personal, 29.05.2015)

Otro principio básico o rector de la valoración de la realización académica en que se notan concepciones diferentes y divergentes es el del origen o del nacimiento de las formas de valoración; es decir, si estas formas debieran surgir del contexto local o del exterior. La divergencia podríamos verla muy bien como un debate entre concepciones, pues una de ellas se manifiesta a favor de un nacimiento más original, autóctono o nativo. Deberíamos regirnos por formas de valoración que hayan surgido de la propia realidad nacional, en lo posible, se sostiene.

Yo creo que es un conflicto que nosotros tenemos en Filosofía y que tiene todo el mundo en ciencias sociales, la universidad y en general el país. Lo que se quiere es estandarizar la forma de evaluar productos y estandarizar significa básicamente asimilarse a ciertos estándares internacionales. Eso es lo que está buscando la universidad, estandarizar esos productos, la evaluación de los productos y que esa estandarización no sea otra cosa sino la asimilación a ciertos estándares internacionales bajo el supuesto de que esos estándares internacionales tienen una mayor experiencia o tienen unos mejores criterios de evaluación que los que podríamos tener nosotros si los creáramos aquí, nosotros mismos. (Entrevistado 40, comunicación personal, 04.09.2015)

Esta visión cuestiona duramente que las formas actuales no sean genuinas y que se sustenten en una creencia de que las formas superiores o más refinadas de valoración solo puedan crearse fuera del país. La crítica se completa con la observación de que la heteronomía de criterios conduce a su inadecuación o no pertinencia, puesto que estos criterios responden a otros propósitos externos, sin relación con la realidad del país. En resumen y analizando, vislumbramos una concepción autonomista y autoctonista sobre el origen de las formas de valoración, y otra, la contraria, que entiende que el tema del origen es un falso problema, falaz, en una época de globalización académica que es sinónimo de conformación de un campo académico global. 
Independientemente de la genealogía de las formas de valoración, está en discusión, también vívidamente, lo que podríamos llamar la matriz de referencia o matriz paradigmática de estas prácticas. Las formas de valoración, ¿deberían fundarse e inspirarse en las ciencias naturales? ¿Deberían las ciencias sociales construir sus propias formas? Este problema, en el discurso de los entrevistados, en verdad alude a otras cuestiones más de fondo, como es el principio básico o general de tolerancia y respeto a la diferencia en que debería estribar toda valoración. Ahora bien, retomando el punto de interés, el del papel paradigmático de las ciencias naturales, lo que atisbamos es la existencia de una concepción más purista y defensora de la singularidad de las ciencias sociales y su realización académica, para la que todo lo que se necesite en estas disciplinas tiene que provenir de ellas mismas y sus atributos característicos. El asunto a subrayar aquí, y esto vuelve a enlazar con el problema de la tolerancia y respeto a la diferencia, es que, para esta concepción, el canon que rige las formas de valoración actual responde a los ideales de las ciencias naturales, pero, como canon que es, se legitima con la anulación de otras identidades, por decirlo de esta manera.

[Hay] algo que repercute de manera directa en las posibilidades de difusión de trabajos de quienes están metidos en este campo de las ciencias humanas. (...) [los] parámetros se definen a partir del trabajo de las ciencias naturales o las ciencias aplicadas. Ambas vertientes son en gran medida opuestas por su forma de trabajo. En ciencias humanas uno más uno no siempre da dos. Otras son las metodologías, otros son los problemas, otros son los impactos, otras son las preocupaciones. Y en este país, al parecer, eso se desconoce, (...) [los] sistemas de clasificación (...) son muy hostiles y desconocen esas formas de trabajo de las ciencias humanas. (Entrevistado 30, comunicación personal, 23.06.2015)

Según los testimonios que atañen a la concepción que llamamos purista, es un canon que se expande y proyecta en desmedro de la otredad, y que halla sustentación en la supuesta superioridad de las ciencias naturales. La concepción discrepante creería que la concepción purista es equívoca porque parte de la errada premisa de la existencia de ciencias en plural y no una sola ciencia. Para esta concepción, un asunto que es tan solo de unos neutrales estándares que se han impuesto, es ideologizado por el purismo, al traducirlo como una cuestión de tolerancia y respeto a las diferencias.

La discrepancia de concepciones se observa, asimismo, en el momento que la purista admite que los procesos de valoración deben efectuarse por evaluadores acreditados y expertos, pero que deben integrar además a otros actores no académicos. Para este sector de los investigadores, las formas actuales de valoración son excesivamente académicas y academicistas. Son, en ese sentido, de un alcance o cobertura muy limitada. Esta concepción pone en el tapete el peso que debería tener 
lo no académico o extraacadémico en los procesos de valoración. Si bien el purismo no ofrece una fórmula respecto de la ponderación de estos dos componentes de las valoraciones, lo que hay es una concepción que quiere hacer evidente la necesidad de combinarlos en la academia. Esta manera de ver las cosas, de la que hemos presentado ampliamente sus preceptos característicos, es un discurso sumamente elaborado, donde advertimos una crítica acentuada al que se denomina academicismo.

Así, el academicismo contemporáneo acarrea varias consecuencias negativas que terminan siendo formas de discriminación y marginación: que ciertos productos o realizaciones no sean reconocidos como legítimos, que determinados estilos de comunicar queden fuera del conjunto de productos dignos de valorar (ensayos, por ejemplo) y que esto fortalezca una comunidad de investigadores con clases de investigador. "Impacto también en coordinación con formas de lucha. Resulta que eso también implica ser leído. O tener impacto e interlocución con movimientos sociales e interlocución con la política pública" (Entrevistado 28, comunicación personal, 10.08.2015).

Las concepciones que pudimos discernir y tipificar se hacen más incompatibles cuando se las coteja en relación con unos asuntos todavía de más calado, como son la adecuación, justicia y sentido de las formas de valoración actual en el mundo de la investigación en las ciencias sociales colombianas. Para la que catalogamos como concepción purista y crítica, el que las formas de valoración no se correspondan con las necesidades reales de la academia, su exotismo, es de por sí algo grave, pero lo es más que esta no correspondencia genere injusticia social y una condición existencial del investigador con inmerecidas e innecesarias tribulaciones resultado de una ambigüedad de valores. El gran reproche de la concepción crítica es que la valoración actual ha trastocado la relación medio-fin, al valorar el producto por el producto, al dejar casi sin importancia lo que algunos llaman el impacto social de la actividad de investigar.

¿Qué pasa con estos informes de investigación en antropología? Son informes, son para investigadores. Y eso sí es una cosa muy complicada de manejar porque eso, como dice mucha gente, eso no se lo lee nadie. Debe haber dos versiones, una que es el informe académico, y otra que es el informe para la persona del común. Pero casi siempre el investigador solo llega al informe técnico y ahí queda. Pero si se logra llegar a unos informes mucho más pensando en la comunidad, el problema es de la publicación: ¿dónde publico yo esto? Eso es donde muchas veces el investigador se limita a casi que a su informe técnico y deja esa otra parte de lado. (Entrevistado 23, comunicación personal, 03.09.2015) 
Podemos finalizar este apartado señalando que las concepciones coexistentes divergen, incluso, respecto del sentido profundo de las formas de valoración actual de la realización académica. En otras palabras, en si estas formas trastocan los medios y los fines, en si valorar sea y deba ser una práctica eminentemente técnica, especializada, y en si deba autonomizarse al punto de transformarse en un sistema clausurado. A esto hay que agregarle la disputa que genera la concepción purista o crítica cuando denuncia que las formas de valoración están contagiadas de criterios empresariales o son estos las que los inspiran. Sobre este tema, la diferencia entre concepciones, que es una diferencia grande en el seno de la comunidad de los investigadores estudiados, se hace mayúscula.

\section{¿Dónde reside el valor de un producto académico o científico?}

¿En qué reside esencialmente el valor de lo que se denomina producto o productos de la actividad académica o científica? ¿En dónde reside su valor? Esta cuestión es una de las más cautivantes de la investigación, no solo por la importancia misma que tiene como asunto complejo y divisor de tipo social, sino porque hace aflorar con más claridad unas concretas concepciones entre los investigadores, dignas de muchísima atención y análisis. Cabe aclarar que las respuestas que presentaremos de los sujetos estudiados se refieren, por lo general, a artículos y libros.

Una primera característica llamativa que surge del análisis de los testimonios es la inmensa capacidad de los investigadores para encontrar aspectos y factores posibles que involucran y que deben involucrar la localización del valor de los productos académicos. Si se hace una lista de lo dicho por ellos, es una relación que excede cualquier creación propia de un solo individuo:

- El valor reside en la novedad del producto, artículo o libro.

- Tendrá más valor si esa novedad tiene un carácter extradisciplinar; es decir, si incorpora elementos de disciplinas distintas a la de la matriz disciplinar del investigador.

- El valor reside en la originalidad.

- Los productos son más valiosos si contienen reflexividad, esa especie de confesión del investigador sobre su incumbencia existencial en la cuestión que la investigación aborda.

- En que tenga algo, necesariamente, de mercancía vendible.

- En que sea útil para la gente en general. 
- En que el producto tenga en la base un proceso de investigación.

- En que ese producto no tenga fallas.

- En que represente o contenga un tema relevante.

- En que se ajuste a la revista o editorial donde se pretende que sea publicado.

- En que tenga buenas citas, si es un libro o un artículo.

- El valor reside en su coherencia metodológica y conceptual.

- En que favorezca el propio crecimiento de su autor.

- En su contribución a la solución de problemas concretos.

- En su aporte teórico.

- Que tenga creatividad.

- Que tenga algo impactante.

- Que tenga algo literario.

- El valor de un producto reposa en su vividez, es decir, en que transmita vida, la vida de su autor, el investigador.

- El valor está en la autenticidad.

- En el pensamiento propio.

- Un producto valioso es aquel que tiene aplicabilidad.

- En que sea pedagógico y que sirva a la pedagogía.

Una primera conclusión rápida es que, siendo tan distintos los aspectos y factores en que reposa esencialmente el valor de un producto, asistimos a un mundo de las ciencias sociales, al menos al mundo que toca a la Universidad de Antioquia y a la Universidad de los Andes, donde no es posible arribar a consenso alguno, si fuera esta la circunstancia hipotética a la que nos enfrentáramos. El hecho de que los investigadores sitúen el valor de los productos en cuestiones tan disimiles daría la impresión de un orden social de las ciencias sociales donde cada cual se siente en la capacidad de profesar preferencia por un único factor determinante del valor, o un orden social más bien inexistente.

Ahora bien, tratando de encontrar patrones y desde ahí reconstruir tipos de concepción, la interpretación anterior se torna, indefectiblemente, parcial. Viéndolo bien, hay ahí unas grandes concepciones, no solo sobre el punto en que estriba el valor de un producto, sino de la investigación en las ciencias sociales, de la identidad de investigador social y su papel en la historia. Podríamos proponer varias maneras de captar estas concepciones. Una sería la que se concentra en lo ingénito o propio de un producto, una especie de concepción inmanentista. Aquí, lo valioso de un producto 
depende o recae en el producto mismo. Es una visión que cree en la autosuficiencia del producto para ser considerado como valioso.

En materia de relaciones internacionales, son explicaciones concretas a problemas concretos científicos. Y ahí es donde le encuentro valor al nivel del convencimiento que me genere una explicación sobre un problema determinado. Qué se yo, cuando te hablan de problemas empíricos determinados. Hablo de cosas como por qué hay Estados que cumplen y Estados que no cumplen con normas de derechos humanos a nivel internacional. ¿Cómo puede explicar uno eso? Y un buen texto, simplemente, me convencería de una hipótesis concreta (Entrevista 36, comunicación personal, 01.09.2015).

Esta concepción inmanentista es una concepción del valor desde las características intrínsecas de los productos académicos, cualidades o propiedades que deberían poder verse y estar en estos. La otra concepción la calificaríamos como una concepción relacional del valor, puesto que no se fija en lo inherente per se, sino en unos aspectos que trascienden al producto mismo y que son establecidos, por así decirlo, desde afuera. Ya no es el producto como tal, sino el producto en relación con unos factores extraproducto, que hacen de esta concepción una perspectiva metavalorativa de los resultados de la actividad académica o de investigación.

Lo que debe ser novedoso tiene que pensarse de otra forma. Por ejemplo, la interdisciplinariedad es un criterio. Ahí, me parece, se evidencia qué es importante. Es decir, que uno como filósofo, que pueda trabajar o hacer un tipo de reflexiones sobre procesos culturales, sobre procesos políticos, sobre prácticas de política, pero que puedan ser interesantes para un antropólogo o que puedan ser interesantes para un historiador. Novedoso, no es solo novedoso en esta comunidad de especialistas, sino que pueda ser algo que resulta novedoso para otras personas, en otras disciplinas que están pensando desde otros ángulos, por ejemplo, prácticas y procesos políticos en los movimientos sociales. (Entrevistado 10, comunicación personal, 21.05.2015)

Con el análisis precedente, rehicimos la lista anterior, ahora clasificada con la nueva tipificación:

\section{Concepción inmanentista:}

- En que el producto tenga en la base un proceso de investigación.

- En que ese producto no tenga fallas.

- En que represente o contenga un tema relevante.

- En que tenga buenas citas, si es un libro o un artículo. 
- El valor reside en su coherencia metodológica y conceptual.

- En que favorezca el propio crecimiento de su autor.

- Que tenga algo literario.

- Que tenga algo impactante.

- En el pensamiento propio.

- En que se ajuste a la revista o editorial donde se pretende que sea publicado.

- El valor está en la autenticidad.

- El valor de un producto reposa en su vividez, es decir en que transmita vida, la vida de su autor, el investigador

\section{Concepción trascendentalista o relacional del valor de un producto:}

- El valor reside en la novedad del producto, artículo o libro.

- Tendrá más valor si esa novedad tiene un carácter extradisciplinar; es decir, si incorpora elementos de disciplinas distintas a la de la matriz disciplinar del investigador.

- El valor reside en la originalidad.

- Los productos son más valiosos si contienen reflexividad, esa especie de confesión del investigador sobre su incumbencia existencial en la cuestión que la investigación aborda.

- En que tenga algo, necesariamente, de mercancía vendible.

- En que sea útil para la gente en general.

- En su contribución a la solución de problemas concretos.

- En su aporte teórico.

- Que tenga creatividad.

- Un producto valioso es aquel que tiene aplicabilidad.

- En que sea pedagógico y que sirva a la pedagogía.

Pero fuimos más específicos en este análisis. Si bien podría caerse en la tentación de caracterizar la primera como la típica concepción académica de las cosas, esa que valora sustancialmente la autonomía de la academia y que cree que lo académico se explica por lo académico y que lo académico se resuelve académicamente, se caería nuevamente en una interpretación parcial. En realidad, en esta primera gran categoría de concepción hay matices que destacar. Vimos grados de inmanentismo, de tal rango que los más extremos parecieran concepciones, paradójicamente, trascendentalistas y no inmanentistas: la autenticidad, la vividez, el propio crecimiento del 
autor del producto, la reflexividad; frente a que no tenga fallas, a que tenga buenas citas, a que estribe en un proceso de investigación. En cuanto a la otra gran categoría de concepciones, la trascendentalista, pareciera suceder algo similar, por tanto, se hace necesaria la matización. En esa concepción está tanto la posición que confiere todo el valor de un producto a unas circunstancias y sujetos externos a la academia (pedagogía, solución de problemas, mercancía vendible, utilidad para la gente en general) como la que les atribuye todo el valor a propiedades como el aporte teórico, la creatividad o la originalidad.

En síntesis, convivirían, no cabe duda, sujetos con horizontes de pensamiento bien contrapuestos en el mundo bajo observación. Lo más significativo, para nosotros, fue constatar la existencia de diversidad en ambas universidades. Pero observamos que no solo se piensa distinto, sino que se busca conservar, defender y fortalecer, por parte de los sujetos, estas perspectivas sobre el valor de los llamados productos, tanto como sobre la razón de ser de la investigación y lo que es y debería ser un investigador social. Interpretando estas controversias, podrían ser luchas por la hegemonía en los predios de las ciencias sociales. Estas concepciones podrían también interpretarse como proyectos sociales y políticos, uno de corte más realista y otro, por decirlo de alguna manera, de corte utopista.

\section{La idea de equidad y su sentido en el nuevo régimen de la ciencia}

Las transformaciones dadas en la estructura institucional de la ciencia y la academia en Colombia, y la forma en la cual los investigadores han experimentado las normas y procedimientos que estas transformaciones han traído consigo han tenido como efecto unas elaboraciones sobre la justicia y equidad de esos cambios. Así lo vimos entre los testimonios.

Muchos de los investigadores que comparten la que denominamos concepción institucionalista, reconocedora de la necesidad del cumplimiento de las normas vigentes, consideran que la modalidad de normalización e institucionalización actual ha generado condiciones de igualdad, pues la profesionalización, regulación, formalización o, como quiera verse, ha sembrado reglas de juego claras y unos tipos de participación académica que permiten acceder a los diferentes recursos científicos, de acuerdo con los intereses, esfuerzos y méritos que tenga el investigador.

La otra concepción, la de la crítica institucional, es la que pone el acento en que los procesos evaluativos vigentes han afectado profundamente las condiciones de trabajo y de participación en los contextos académicos. Esta perspectiva denuncia 
una marginación de métodos, disciplinas y experiencias singulares individuales. Los investigadores que han desarrollado tal percepción asocian esta marginación a la implementación de un modelo de evaluación centrado en las formas y lógicas de producción de las ciencias naturales, que presume la aplicación legítima de este canon a las ciencias sociales y humanas. El resultado es una situación de imposición de unos lenguajes y métodos que excluyen otros tipos de realización que pueden contar con atributos de gran calidad e impacto, pero que, por no responder a los términos del régimen, son exiliados como producciones apóstatas. Todo ello margina a investigadores y disciplinas que no se ciñen a sus parámetros.

El sector de académicos críticos de la institucionalidad dada quiere poner en evidencia la dificultad que tendría el régimen actual para reconocer las realizaciones que, siendo de calidad y rigor, no se someten o adaptan a los nuevos requerimientos, como por ejemplo de las revistas, que exigen unas determinadas estructuras argumentativas o el tratamiento de unos particulares temas de investigación. Los representantes de esta perspectiva evocan experiencias de trabajo (artículos, libros) que reciben evaluaciones dispares, de gran incomprensión, en las que los criterios, argumentos, enfoques y teorías que se aplican no necesariamente responden a los tenidos en cuenta en la elaboración de esos productos. Una consecuencia negativa de las modalidades de evaluación imperantes son los rechazos, que no tienen justificación porque resultan de una gran falta de empatía. El rechazo desestimula el interés por publicar, así lo manifiestan. Estos investigadores consideran, asimismo, que dentro del régimen científico actual ciertas disciplinas son discriminadas según las cualidades que posean de ser funcionales o más funcionales al sistema. La mayor funcionalidad tiene recompensa, como tener acceso a recursos, becas, subvenciones.

\footnotetext{
Aunque es cierto que es complejo tener un solo estándar para todos, pareciera que los estándares los hacen pensando desde ciertas disciplinas y no desde otras disciplinas, como son las ciencias sociales. Pensando desde este punto de vista, es injusto. No es lo mismo catalogar la producción en psicología que en historia, geografía. Si logramos esa forma de estandarizar, vamos a caer siempre en que hay cierta injusticia. (Entrevistado 28, comunicación personal, 10.08.2015)
}

Sobre la inequidad tampoco pudimos percibir unas tendencias que nos permitieran inferir una concepción más central entre el conjunto de los académicos estudiados, de ambas universidades. No vimos tendencias centrales sobre el tema de la inequidad a nivel laboral, por ejemplo. La posición crítica y la institucionalista difieren mucho, porque, para la primera, las realizaciones académicas que no responden a los criterios privilegiados reciben un menor reconocimiento, independientemente del aporte, calidad o rigor con el cual estas hayan sido creadas, inequidad que se 
refleja en salarios y escalafones, puesto que la burocracia ha creado un sistema para recompensar en razón del tipo de realización.

\section{Reflexiones finales}

Este trabajo cumple con el objetivo de desarrollar una exploración interpretativa sobre lo que piensan, perciben o creen (concepciones) los sujetos observados sobre su contexto inmediato de acción y en referencia a los términos de lo que llamamos régimen actual de la ciencia y la academia en Colombia. Es una dilucidación que aporta elementos para el abordaje de la subjetividad de los investigadores sociales y las formas en que proyectan el ejercicio de su labor. Ciertamente, en una próxima fase de esta misma investigación, habrá que conectar las concepciones reconstruidas con las condiciones de la existencia social, en términos de Mannheim (2004). Es muy probable, como afirma su clásica teoría, pero también el llamado programa fuerte de sociología y toda la sociología del conocimiento, que las diferencias de concepción encontradas respondan a posiciones en el espacio académico, posiciones que pertenecen a estructuras sociales de clasificación.

Por su parte, el actual entramado institucional de la ciencia colombiana parece haber ocasionado desplazamientos en las prácticas y concepciones de la realización académica e investigativa. Aquellas prácticas y concepciones que caracterizaban el trabajo académico en las ciencias sociales han virado, debido a las reglas de juego imperante, hacia unas maneras de proceder cognitivas, discursivas y ejecutoras más propias de una concepción empresarial.

Pero estos investigadores de las ciencias sociales de las dos universidades estudiadas no piensan igual; sostienen y desarrollan concepciones distintas sobre, aparentemente, los mismos marcos sociales o contextos históricos: hay agrupamientos de concepciones y tipos de concepción. En términos generales, estas concepciones transitan entre una aceptación de los cambios, que viene acompañada por un discurso sobre su necesidad, pertinencia, justificación y valor; y una negación crítica, que viene apoyada por toda una concepción distinta sobre lo que es y debe ser la academia, la ciencia y la realización académica.

Además, hay preocupación respecto a las condiciones del desarrollo de la actividad académica. Los sujetos vistos avizoran trasformaciones en las prácticas de la investigación social, debido a la acogida de unos modelos normativos y técnicos de proceder que homogenizan la actividad académica, y donde se perciben unas restricciones de la diversidad, libertad y creatividad de pensamiento y acción. Estos modelos favorecen la implantación de lógicas internacionales y desfavorecen la constitución de una comunidad académica local. 
Ciertamente, desde la perspectiva de las concepciones que desarrollamos en nuestra investigación, el mundo de la academia resulta ser más diverso de lo que solemos pensar y un espacio donde los influjos del entorno parece que difícilmente se reflejan mecánicamente sobre el pensamiento de los sujetos. Así, existe diversidad respecto de los elementos que le otorgan valor a las expresiones de la realización académica. De hecho, es muy difícil encontrar criterios valorativos consensuales y, también, son diversos los contenidos valorativos asociados a proyectos políticos, sociales y académicos.

Finalmente, los modelos de evaluación y la instauración de una estructura de acreditación burocrática parecen haber alterado las prácticas de investigación y las maneras de pensar de un sector de los académicos, haciendo que tiendan a la racionalización al extremo, imponiendo como finalidad per se el hecho mismo de producir y conseguir una acreditación y reconocimiento dentro del exclusivo mundo de la academia, solamente. Esto es reconocido por los investigadores en general, pero, asimismo, es una de las cuestiones sobre las cuales las concepciones existentes parecen ser más disimiles.

\section{Referencias}

Brown, Mark (2009). Science in Democracy. Expertise, Institutions and Representation. London: MIT Press.

Callon, Michel; Courtial, Jean-Pierre; Penan, Herve (1995). Cienciometría. El estudio cuantitativo de la actividad científica: de la bibliometría a la vigilancia tecnológica. Girón: Trea.

Cathalifaud, Marcelo Arnold (2012). Entre el universalismo y el relativismo. Reposicionamiento de las ciencias sociales latinoamericanas. Civitas, 12(1), 9-19.

Consejo Nacional de Política Económica y Social (2015). Política nacional de ciencia, tecnología e innovación, 2015-2025. Bogotá: Departamento Nacional de Planeación.

De Sousa-Santos, Boaventura (2010). Para descolonizar Occidente. Más allá del pensamiento abismal. Buenos Aires: Clacso.

De Sousa-Santos, Boaventura (2011). Una epistemología del sur. La reinvención del conocimiento y la emancipación social. México: Siglo XXI/Clacso.

Etzkowitz, Henry; Leydesdorff, Loet (1997). Universities and the Global Knowledge Economy: A Triple Helix of University-Industry-Government Relations. London: Pinter. 
Gill, Rosalind (2014). Academics, Cultural Workers and Critical Labour Studies. Journal of Cultural Economy, 7(1), 12-30.

Gómez-Morales, Yuri Jack (2015). Usos y abusos de la bibliometría. Revista Colombiana de Antropología, 51(1), 291-307.

Gómez-Morales, Yuri Jack (2017). El baile de los que sobran: cambio cultural y evaluación académica. Revista Colombiana de Antropología, 53(2), 15-25.

Gómez-Morales, Yuri Jack (2018). Abuso de las medidas y medidas abusivas. Crítica al pensamiento bibliométrico hegemónico. Anuario Colombiano de Historia Socialy de la Cultura, 45(1), 269-290.

Guzmán-Tovar, César (2019). Las experiencias de aceleración en investigadores sociales de América Latina. Sociológica, 97(34), 115-144.

Jiménez, Absalón (2015). Colciencias, su devenir e impacto en los grupos de investigación de la Facultad de Ciencias y Educación de la UDFJC. Revista Científica, 22, 7-18.

Kreimer, Pablo (2006). ¿Dependientes o integrados? La ciencia latinoamericana y la nueva división internacional del trabajo. Nómadas, 24, 197-213.

Kreimer, Pablo (2011). La evaluación de la actividad científica: desde la indagación sociológica a la burocratización. Dilemas actuales. Propuesta Educativa, 20(36), 59-77.

Lux, Martha; Pérez, Ana (2017). Reflexiones sobre la producción, circulación y uso de las publicaciones académicas en las ciencias sociales. Anuario Colombiano de Historia Social $y$ de la Cultura, 44(1), 125-143.

Mannheim, Karl (2004). Ideología y utopía. Introducción a la sociología del conocimiento. México: FCE.

Marcuse, Herbert (1993). El hombre unidimensional. Ensayo sobre la ideología de la sociedad avanzada. Barcelona: Planeta-Agostini.

Masías, Rodolfo (2014). Los investigadores sociales en Colombia: producción, productividad y diferenciación social. Revista Colombiana de Sociología, 37(1), 123-156.

Nupia, Carlos (2013). Orígenes de la política científica y tecnológica en Colombia. Colciencias y su papel en la transferencia de modelo internacional de "política científica". En Colciencias cuarenta años entre la legitimidad, la normatividad y la práctica (pp. 121-178). Bogotá: Observatorio de Ciencia y Tecnología/Universidad Nacional de Colombia/Universidad del Rosario.

Santos, José (2012). Tiranía del paper. Imposición institucional de un tipo discursivo. Revista Chilena de Literatura, 82, 197-217. 
Santos, José (2015). Saberes académicos: de la producción textual a la creación de conocimiento. Literatura: Teoría, Historia, Crítica, 17(2), 97-112. http://doi.org/10.15446

Vasen, Federico; Lujano-Vilchis, Ivonne (2017). Sistemas nacionales de clasificación de revistas científicas en América Latina: tendencias recientes e implicaciones para la evaluación académica en ciencias sociales. Revista Mexicana de Ciencias Sociales y Políticas, 62(231), 199-228.

Vessuri, Hebe (2013). ¿Quién es el científico social en el siglo XXI? Comentarios desde los contextos académicos y aplicados y desde la corriente principal y la periferia. Sociológica, 28(79), 201-231.

Villaveces, José Luis; Forero-Pineda, Clemente (2007). Cincuenta años de ciencia en Colombia, 1955-2005. En Fundación Alejandro Ángel Escobar, 50 años (pp.97-133), editado por Clemente Forero-Pineda. Bogotá: Fundación Alejandro Ángel Escobar.

Wallerstein, Immanuel (1999). Abrir las ciencias sociales. Informe de la Comisión Gulbenkian para la reestructuración de las ciencias sociales. México: Siglo XXI.

Wallerstein, Immanuel (2005). Las incertidumbres del saber. Barcelona: Gedisa. 